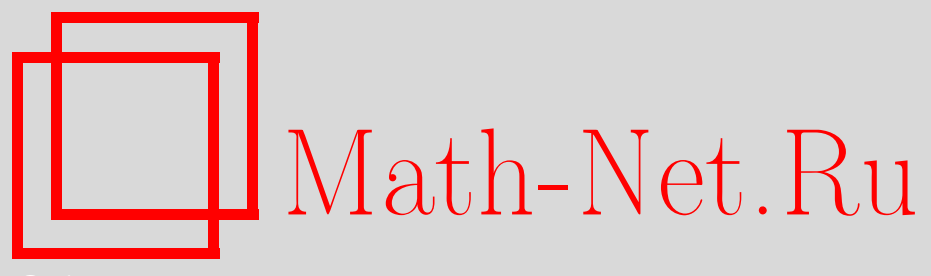

В. Ф. Москва, А. М. Райгородский, Новые нижние оценки чисел независимости графов расстояний с вершинами в $\{-1,0,1\}^{n}$, Матем. заметки, 2011, том 89, выпуск $2,319-320$

DOI: https://doi.org/10.4213/mzm8940

Использование Общероссийского математического портала Math-Net.Ru подразумевает, что вы прочитали и согласны с пользовательским соглашением http: //www. mathnet.ru/rus/agreement

Параметры загрузки:

IP: 3.80 .181 .102

26 апреля 2023 г., 17:29:42

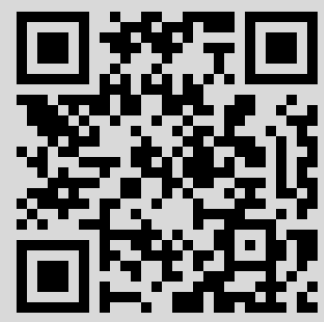




\section{Новые нижние оценки чисел независимости графов расстояний с вершинами в $\{-1,0,1\}^{n}$}

В. Ф. Москва, А. М. Райгородский

1. Введение и формулировка результата. Пусть $n=2 k, k \in \mathbb{N}$. Рассмотрим граф $G_{n}=\left(V_{n}, E_{n}\right)$, у которого

$$
\begin{gathered}
V_{n}=\left\{\mathbf{x}=\left(x_{1}, \ldots, x_{n}\right): x_{i} \in\{-1,0,1\},\left|\left\{i: x_{i}= \pm 1\right\}\right|=\left|\left\{i: x_{i}=0\right\}\right|=k\right\}, \\
E_{n}=\left\{\{\mathbf{x}, \mathbf{y}\}: \mathbf{x}, \mathbf{y} \in V_{n},|\mathbf{x}-\mathbf{y}|=\sqrt{2 k}\right\} .
\end{gathered}
$$

Будем считать, что в каждом векторе из $V_{n}$ первая ненулевая координата равна 1 . Таким образом, $\left|V_{n}\right|=C_{n}^{k} 2^{k-1}$.

Граф $G_{n}$ играет исключительно важную роль в проблемах Борсука и Нелсона-Эрдеша-Хадвигера (см. [1]-[6]). В частности, вопрос об отыскании числа независимости

$$
\alpha\left(G_{n}\right)=\max \left\{|W|: W \subseteq V_{n},\{\mathbf{x}, \mathbf{y}\} \notin E_{n}, \mathbf{x}, \mathbf{y} \in W\right\}
$$

такого графа крайне интересен и по-прежнему открыт.

Впервые этот вопрос возник в работе [3]. Затем он обсуждался в книге [2]. Наконец, в статьях [7]-[9] были предложены новые нетривиальные подходы к проблеме, которые позволили получить как асимптотические верхние и нижние оценки, так и их аналоги в малых размерностях. Сейчас нам будут более интересны асимптотические результаты, к ним мы и перейдем.

В работах [7], [8] доказана

Теорема 1. Имеет место оценка $\alpha\left(G_{n}\right) \geqslant(2.241 \ldots+o(1))^{n}$.

В свою очередь, наилучшая известная верхняя оценка (см. [8]) дается в теореме 2.

Теорема 2. Имеет место оценка $\alpha\left(G_{n}\right) \leqslant(2.462 \ldots+o(1))^{n}$.

Очевидно, что зазор довольно велик. В книге [2] предложена многопараметрическая конструкция, которая при правильном подборе параметров, с большой вероятностью оптимальна. Проведя соответствующую оптимизацию, мы пришли к следующему утверждению.

Теорема 3. Имеет место оченка $\alpha\left(G_{n}\right) \geqslant(2.264 \ldots+o(1))^{n}$.

ГиПотезА 1. Имеет место равенство $\alpha\left(G_{n}\right)=(2.264 \ldots+o(1))^{n}$.

Гипотеза 1 имеет ряд важных следствий, о которых мы скажем в п. 2 данной заметки.

2. Возможные приложения в проблемах Борсука и Нелсона-Эрдеша-Хадвигера. Проблема Борсука состоит в отыскании минимального числа $f(n)$ частей меньшего диаметра, на которые разбивается произвольное ограниченное неодноточечное множество в $\mathbb{R}^{n}$ (см. [1]-[6]). Проблема Нелсона-Эрдеша-Хадвигера сводится к поиску величины $\chi\left(\mathbb{R}^{n}\right)$, называемой хроматическим числом пространства и равной наименьшему количеству цветов, в которые можно так покрасить все точки пространства, чтобы между одноцветными точками не было расстояния 1 (см. [1], [2]).

В предположении справедливости гипотезы 1 можно обосновать следующие две теоремы.

Работа выполнена при финансовой поддержке Российского фонда фундаментальных исследований (грант № 09-01-00294), гранта Президента РФ (грант № МД-8390.2010.1), программы "Ведущие научные школы" (грант № НШ-8784.2010.1).

(C) В.Ф. Москва, А. М. РАйгородский, 2011 
Теорема 4. Имеет место оценка $\chi\left(\mathbb{R}^{n}\right) \geqslant(1.249 \ldots+o(1))^{n}$.

Теорема 5. Имеет место оценка $f(n) \geqslant(1.369 \ldots+o(1))^{\sqrt{n}}$.

Обе эти теоремы значительно уточняют известные неравенства

$$
\chi\left(\mathbb{R}^{n}\right) \geqslant(1.239 \ldots+o(1))^{n}, \quad f(n) \geqslant(1.225 \ldots+o(1))^{\sqrt{n}}
$$

(см. [1]).

ДОКАЗАТЕЛЬСТво ТЕОРЕМЫ 4 совсем простое:

$$
\chi\left(\mathbb{R}^{n}\right) \geqslant \chi\left(G_{n}\right) \geqslant \frac{\left|V_{n}\right|}{\alpha\left(G_{n}\right)}=\frac{(2 \sqrt{2}+o(1))^{n}}{(2.264 \ldots+o(1))^{n}}=(1.249 \ldots+o(1))^{n} .
$$

Доказательство теоремы 5 требует более сложного рассуждения, которое стандартно (см. [1], [2]) и выходит за рамки этого краткого сообщения.

\section{СПИСОК ЦИТИРОВАННОЙ ЛИТЕРАТУРЫ}

[1] А. М. Райгородский, УМH, 56:1 (2001), 107-146. [2] А. М. Райгородский, Линейно-алгебрачческий метод в комбинаторике, МЦНМО, М., 2007. [3] A. M. Raigorodskii, Surveys in Contemporary Mathematics, London Math. Soc. Lecture Note Ser., 347, Cambridge Univ. Press, Cambridge, 2008, 202-247. [4] В.Г. Болтянский, И.Ц. Гохберг, Теоремь и задачи комбинаторной геометрии, Наука, М., 1965. [5] V.G. Boltyanski, H. Martini, P.S. Soltan, Excursions into Combinatorial Geometry, Universitext, Springer, Berlin, 1997. [6] P. Brass, W. Moser, J. Pach, Research Problems in Discrete Geometry, Springer, New York, 2005. [7] В. К. Любимов, А. М. Райгородский, Докл. РАН, 427:4 (2009), 458-460. [8] А. Э. Гутерман, В. К. Любимов, А. М. Райгородский, А. С. Усачев, "О числах независимости графов расстояний с вершинами в $\{-1,0,1\}^{n}$ : оценки, гипотезы и приложения к задачам Борсука и Нелсона-Эрдеша-Хадвигера”, Итоги науки и техн. Сер. Соврем. мат. и ее прил. Темат. обз. (в печати). [9] А.Э. Гутерман, В. К. Любимов, А. М. Райгородский, А. С. Усачев, Матем. заметки, 86:5 (2009), 794-796.

В. $\Phi$. Москва

Поступило

Московский физико-технический институт

10.06.2010 (государственный университет)

\section{А. М. Райгородский}

Московский государственный университет им. М. В. Ломоносова

E-mail: mraigor@yandex.ru 